\title{
Erratum
}

\section{Chemical abundance patterns - fingerprints of nucleosynthesis in the first stars}

\author{
T. Karlsson and B. Gustafsson \\ Department of Astronomy and Space Physics, Uppsala Astronomical Observatory, Box 515, \\ 75120 Uppsala, Sweden
}

A\&A, 379, 461-481 (2001), DOI: 10.1051/0004-6361:20011217

Figures 17 and 18 on page 478 were misprinted. The metallicity of the stars, measured by [Fe/H], was wrongly indicated and one star was missing. The corrected figures are shown below. No conclusions are altered.

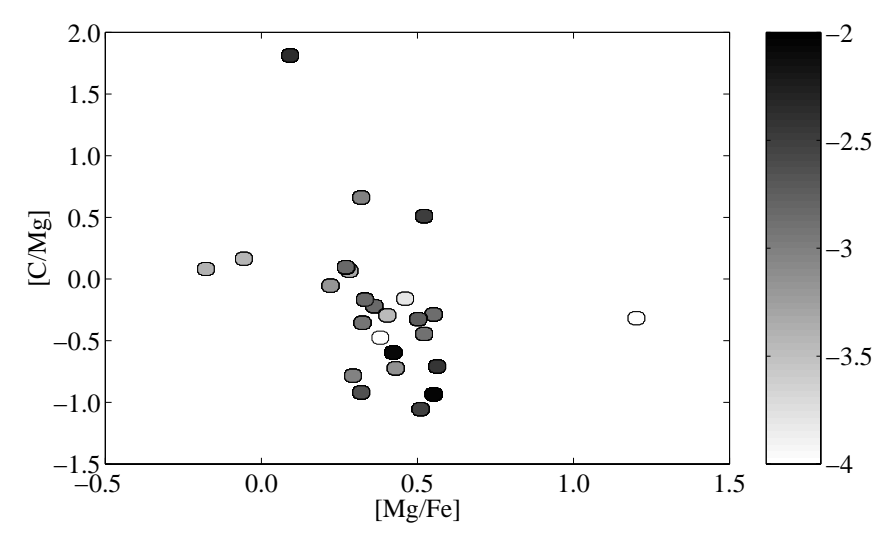

Fig. 17. Observed abundances of stars from McWilliam et al. (1995) displayed in the $[\mathrm{Mg} / \mathrm{Fe}]-[\mathrm{C} / \mathrm{Mg}]$ plane (cf. Figs. 5a and d). The shading according to the bar on the right indicates the metallicity measured by $[\mathrm{Fe} / \mathrm{H}]$. Stars with $B-V>0.80$ are removed from the sample in order to minimize the effect of carbon depletion in the red giants. There are too few stars to allow detection of any patterns although there seems to be a large, asymmetric dispersion.

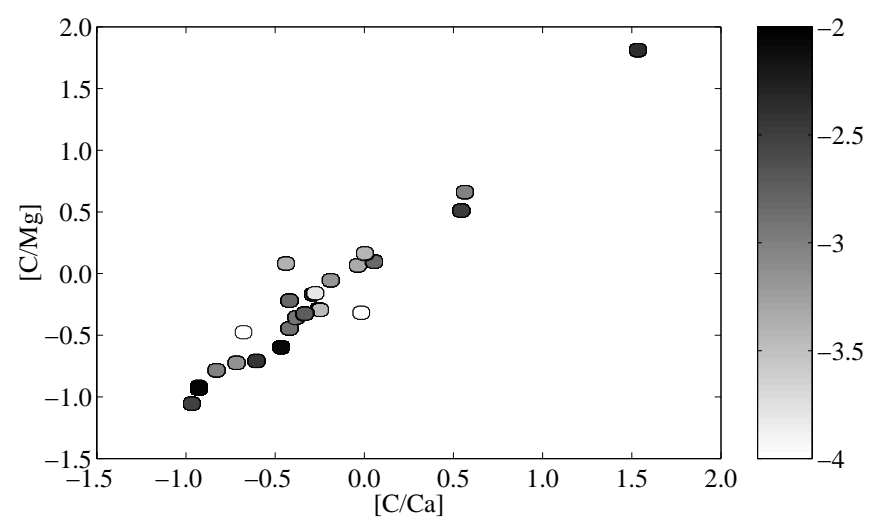

Fig. 18. Abundances of stars from the sample of McWilliam et al. (1995) plotted in the $[\mathrm{C} / \mathrm{Ca}]-[\mathrm{C} / \mathrm{Mg}]$ plane. As for Fig. 17, the symbols are shaded according to the stellar metallicity. Stars with $B-V>0.80$ have been removed from the sample. Note that the observed stars form a tight relation with a slope close to one.

Send offprint requests to: T. Karlsson, e-mail: Torgny.Karlsson@astro.uu.se 